bounded and self-adjoint in the space of all k-periodic sequences. Then a special functional was constructed, the critical points of which in this space are solutions of the original equation. A Gateaux derivative of this functional is found. Next we consider the Nehari manifold for a given variational problem, which is a set of nontrivial critical points of a constructed functional in the space of k-periodic sequences. It is shown that this manifold is a non-empty and closed subset of a given space. In addition, the corresponding minimization problem is considered and it is shown that this problem has a solution in the Nehari manifold. Consequently, under these conditions the original equation has nontrivial periodic solutions. Finally, due to the fact that saturable nonlinearity satisfies these conditions, the existence of two nontrivial standing waves with k-periodic amplitude for a discrete nonlinear Schrödinger equation with saturable nonlinearity on a two-dimensional lattice is established. The results of this paper are the distribution of already known results for discrete nonlinear Schrödinger equations on 1D and 2D-lattices.

Key words: discrete nonlinear Schrödinger equation, 2D-lattice, standing waves, critical points, Nehari manifold, saturable nonlinearity.

Отримано: 14.11.2018

UDC 519.6

DOI: $10.32626 / 2308-5878.2018-18.14-24$

A. Ya. Bomba, Doct. of Techn. Sciences, M. V. Boichura

Rivne State Humanitarian University, Rivne

\title{
NUMERICAL COMPLEX ANALYSIS METHOD FOR PARAMETERS IDENTIFICATION OF ANISOTROPIC MEDIA USING APPLIED QUASIPOTENTIAL TOMOGRAPHIC DATA. PART 1: PROBLEM STATEMENT AND ITS APPROXIMATION
}

The approach to the solving of gradient problems of parameters identification of quasiideal fields with using applied quasipotential tomographic data based on numerical complex analysis methods is transferred to cases of anisotropic media. We, similar to the existing works of world scientists, some additional information about the nature of the distribution of conductivity inside the domain (research object) is considered a priori known. However, in opposite to the traditional approaches to the statement and solving the problems of electrical impedance tomography, we set the local velocities distribution of a substance (liquid, current) in addition to the averaged potential at the contact sections of plate and body and at other sections (stream lines), we set the potential distribution (according to experimental data, which we approximate using splines, Bezier curves, etc.). Generation of initial data at the boundary of the investigated object is carried out in accordance with the polar model of current injection and a given sum of eigenvalues of the conductivity tensor of 
the medium. The presence of this kind of data greatly accelerates the process of further solving the problem, which is convenient, in particular, when verifying the method that developed by authors. The corresponding problem is reduced to the iterative solving of a series of problems for the Laplace type equations, where instead of «boundary numerical analogues of the Cauchy-Riemann type equations» appear the ratio of quasiorthogonality with using special types of optimization conditions. In particular: the minimizing functional is constructed by taking into account the Cauchy-Riemann type conditions, the relation between eigenvalues of corresponding anisotropy tensor and also regularizing term; the condition-restriction is built based on ellipticity conditions.

Key words: applied quasipotential tomography, quasiconformal mappings, anisotropy, identification, nonlinear problems.

Introduction. As it is known [1-5], the image reconstruction of an isotropic conductivity coefficient that based on the applied quasipotential tomography (AQT) requires the imposition of a large number of conditions at the domain bound, and also the structure of the corresponding medium. It turns out that in the general case (in contrast to some specific [6]) of anisotropy, it is necessary to set much more information about the conductivity distribution for its parameter identification [1,7-12]. This, obviously, weakens the correctness of the problem in comparison with the isotropy. And, consequently, it requires (in comparison with, for example, [5]) the necessity of using a regularizing functional, in particular the Tikhonov type $[1,3,4,9,11]$. The ways to apply additional data about the conductivity tensor (CT), depending on the information type about it are proposed in a number of papers [6-9, 12]. In particular, several options for specifying information about eigenvectors offer in [8]. The regularization procedure was adapted in such a way that the additional data were taken into account as much as possible [9]. A new visual CT representation, in which it is easy to understand its distribution, is given in [6].

On the other hand, today a promising methodology for identifying the conductivity coefficient using AQT data, according to which the solving of the sequence of so-called analysis and synthesis problems is reduced to the alternate application of numerical quasiconformal mappings methods and the parameters identification of the conductivity of the medium, respectively $[4,5]$ is developed. In this paper we discuss the transfer of this methodology to the case of the parameter identification of anisotropic media. At the same time, the additional information is the dependency between eigenvalues of CT.

In this case, the practical application of the results of this research can take place in a number of branches of science and technology (see, for example [1, 9-13]). In particular, in medicine, the object of this kind of research may be the medium with fibrous or layered areas (which includes muscles, bones, etc.), in which there is a stream of non-spherical particles (for example, red blood cells), and in geology, with areas of soil layer compression. 
Parameters identification problem of anisotropic mediums using AQT data. Consider quasiideal processes of particles movement (in particular, fluids, electrical currents) in a single-connected curvilinear domain (horizontal anisotropic formation, anisotropic plate - the tomographical crosssection) $G_{z}$ (Fig. 1, a), bounded by a smooth closed curve $\partial G_{z}=\{(x, y)$ : $x=\tilde{x}(\tau), \quad y=\tilde{y}(\tau), \quad \tilde{x}(0)=\tilde{x}(2 \pi)=\tilde{x}_{0}, \quad \tilde{y}(0)=\tilde{y}(2 \pi)=\tilde{y}_{0}, \quad 0 \leq \tau \leq 2 \pi$, where $\tilde{x}(\tau), \tilde{y}(\tau)$ are defined continuosly differentiated functions, $O\left(\tilde{x}_{0}, \tilde{y}_{0}\right)$ is given an initial starting point $\}$, generated by the existence of potential differences $\varphi_{*}^{(p)}, \varphi^{*(p)}\left(\varphi^{*(p)}>\varphi_{*}^{(p)}\right)$, which are given on selected equipotential lines $A_{p} B_{p}$ and $C_{p} D_{p}$, where $A_{p}, B_{p}, C_{p}, D_{p}$ are marked points on the $\partial G_{z} ; \quad p=1,2, \ldots$ is injection number (see for example $[3-5,8,11]) ; B_{p} C_{p}$ and $A_{p} D_{p}$ are impermeable boundary flow lines. We model a current injection through tomographic cross-section, similarly to [4, 5], by using sets of values $\left\{\tau_{A}^{(p)}, \tau_{B}^{(p)}, \tau_{C}^{(p)}, \tau_{D}^{(p)}\right\}$, corresponding to which

$$
\begin{aligned}
& A_{p}=\left(\tilde{x}\left(\tau_{A}^{(p)}\right), \tilde{y}\left(\tau_{A}^{(p)}\right)\right), B_{p}=\left(\tilde{x}\left(\tau_{B}^{(p)}\right), \tilde{y}\left(\tau_{B}^{(p)}\right)\right), \\
& C_{p}=\left(\tilde{x}\left(\tau_{C}^{(p)}\right), \tilde{y}\left(\tau_{C}^{(p)}\right)\right), D_{p}=\left(\tilde{x}\left(\tau_{D}^{(p)}\right), \tilde{y}\left(\tau_{D}^{(p)}\right)\right) .
\end{aligned}
$$

We denote the corresponding for current injection bound of domain $G_{z}$ with given four marked points by $\partial G_{z}^{(p)}\left(z^{(p)}=x^{(p)}+i y^{(p)}\right)$.

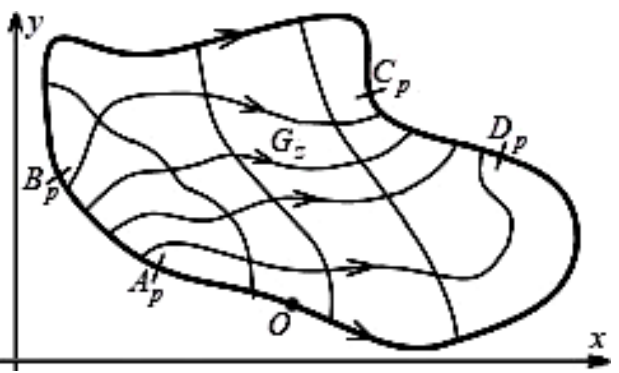

a)

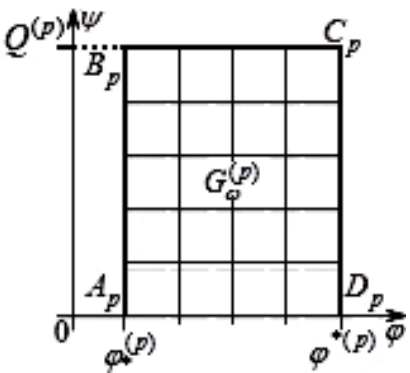

b)

Fig. 1. Tomographical cross-section $G_{z}(a)$ and corresponding complex quasipotential domains $G_{\omega}^{(p)}$ (b)

As a mathematical model of AQT [1] we use, similarly to [4, 5, 14], systems of differential equations in partial derivatives which connect mutually quasicomplex conjugate quasipotentials $\varphi=\varphi^{(p)}(x, y)$ of density $\operatorname{\sigma grad} \varphi^{(p)}(x, y)$ and stream functions $\psi^{(p)}=\psi^{(p)}(x, y)$ when have place 
CT $\sigma=\left(\sigma_{\alpha \beta}(x, y, \ldots)\right)_{\alpha, \beta=1,2}$ identification under corresponding boundary conditions:

$$
\begin{gathered}
\left(\sigma_{11} \varphi_{x}^{(p)}+\sigma_{12} \varphi_{y}^{(p)}\right)_{x}^{\prime}+\left(\sigma_{21} \varphi_{x}^{(p)}+\sigma_{22} \varphi_{y}^{\prime(p)}\right)_{y}^{\prime}=0 ; \\
\left.\varphi^{(p)}\right|_{A_{p} B_{p}}=\varphi_{*}^{(p)},\left.\varphi^{(p)}\right|_{C_{p} D_{p}}=\varphi^{*(p)},\left.|\vec{j}(p)|\right|_{B_{p} C_{p} \cup A_{p} D_{p}}=0 ; \\
\left.\varphi^{(p)}(M)\right|_{B_{p} C_{p}}=\bar{\varphi}^{(p)}(M),\left.\quad \varphi^{(p)}(M)\right|_{A_{p} D_{p}}=\underline{\varphi}^{(p)}(M), \\
\left.\left|\vec{j}^{(p)}(M)\right|\right|_{A_{p} B_{p}}=\Psi_{*}^{(p)}(M),\left|\vec{j}^{(p)}(M)\right|_{C_{p} D_{p}}=\Psi^{*(p)}(M) ; \\
\sigma_{11}+\sigma_{22}=\lambda_{1}+\lambda_{2}=\lambda .
\end{gathered}
$$

Here (1) is a consequence of the linear law of motion such as Ohm, Darcy etc. $\vec{j}^{(p)}=\sigma \operatorname{grad} \varphi^{(p)}$ and the equation of continuity $\operatorname{div} \vec{j}^{(p)}=0[1-8$, $10,13] ; \sigma_{\alpha \beta}=\sigma_{\alpha \beta}(x, y, \ldots)$ are limited continuously differentiated in domain $G_{z}^{(p)}$ functions, which characterize conductivity and anisotropy of medium; $\lambda=\lambda(x, y)$ is given distribution of the sum of eigenvalues $\lambda_{1}$, $\lambda_{2}$ of corresponding to (1) matrix, that equivalent to the sum of diagonal elements of given tensor [14]; $\vec{n}$ is unit vector of outer normal; $M$ is a running point of the corresponding curve. The functions

$$
\begin{gathered}
\bar{\varphi}^{(p)}(M)=\bar{\varphi}^{(p)}(\tau, \ldots) \quad\left(\tau_{C}^{(p)} \leq \tau \leq \tau_{B}^{(p)}\right), \quad \underline{\varphi}^{(p)}(M)=\underline{\varphi}^{(p)}(\tau, \ldots) \\
\left(\tau_{A}^{(p)} \leq \tau \leq \tau_{D}^{(p)}\right), \quad \Psi_{*}^{(p)}(M)=\Psi_{*}^{(p)}(\tau, \ldots) \quad\left(\tau_{B}^{(p)} \leq \tau \leq \tau_{A}^{(p)}\right), \\
\Psi^{*(p)}(M)=\Psi^{*(p)}(\tau, \ldots) \quad\left(\tau_{D}^{(p)} \leq \tau \leq \tau_{C}^{(p)}\right)
\end{gathered}
$$

can be built by the interpolation of the experimentally received their values $\bar{\varphi}_{\bar{i}^{(p)}}^{(p)}, \quad \underline{\varphi}_{\underline{i}^{(p)}}^{(p)}, \quad \Psi_{*_{j_{*}^{*}}^{(p)}}^{(p)}, \quad \Psi_{j^{(p)}}^{*(p)}$ having some $\tau=\bar{\tau}_{\bar{i}^{(p)}}^{(p)}, \quad \tau=\underline{\tau}_{\underline{\tau}^{(p)}}^{(p)}, \quad \tau=\tau_{*_{j_{*}^{*}}^{(p)}}^{(p)}$, $\tau=\tau_{j^{*(p)}}^{*(p)}$ on sections $B_{p} C_{p}, A_{p} D_{p}, A_{p} B_{p}, C_{p} D_{p}$, respectively

$$
\begin{gathered}
\left(\varphi_{*}^{(p)} \leq \underline{\varphi}_{\underline{i}^{(p)}}^{(p)} \leq \varphi^{*(p)}, \quad \varphi_{*}^{(p)} \leq \bar{\varphi}_{\bar{i}^{(p)}}^{(p)} \leq \varphi^{*(p)}, \quad \Psi_{*_{j_{*}}^{(p)}}^{(p)}>0,\right. \\
\Psi_{j^{*(p)}}^{*(p)}>0, \quad 0 \leq \bar{i}^{(p)} \leq \bar{m}^{*(p)}+1,0 \leq \underline{i}^{(p)} \leq \underline{m}_{*}^{(p)}+1, \\
\left.0 \leq j_{*}^{(p)} \leq n_{*}^{(p)}+1, \quad 0 \leq j^{*(p)} \leq n^{*(p)}+1\right)
\end{gathered}
$$

as in [5]. We search the CT components as:

$$
\begin{aligned}
& \sigma_{11}=\sigma_{11}\left(x^{(p)}, y^{(p)}, a_{s_{a}, 0}, \ldots, a_{0, s_{a}}\right)=\sum_{k_{a}, r_{a}=0}^{s_{a}, k_{a}} a_{k_{a}-r_{a}, r_{a}} x^{(p) k_{a}-r_{a}} y^{(p) r_{a}}, \\
& \sigma_{22}=\sigma_{22}\left(x^{(p)}, y^{(p)}, b_{s_{b}, 0}, \ldots, b_{0, s_{b}}\right)=\sum_{k_{b}, r_{b}=0}^{s_{b}, k_{b}} b_{k_{b}-r_{b}, r_{b}} x^{(p) k_{b}-r_{b}} y^{(p) r_{b}},
\end{aligned}
$$




$$
\begin{gathered}
\sigma_{12}=\sigma_{12}\left(x^{(p)}, y^{(p)}, c_{s_{c}, 0}, \ldots, c_{0, s_{c}}\right)=\sum_{k_{c}, r_{c}=0}^{s_{c}, k_{c}} c_{k_{c}-r_{c}, r_{c}} x^{(p) k_{c}-r_{c}} y^{(p) r_{c}}, \\
\sigma_{21}=\sigma_{12},
\end{gathered}
$$

where $\quad a_{k_{a}-r_{a}, r_{a}}, \quad b_{k_{b}-r_{b}, r_{b}}, \quad c_{k_{c}-r_{c}, r_{c}} \quad\left(k_{a}=0, \ldots, s_{a}, \quad r_{a}=0, \ldots, k_{a}\right.$, $\left.k_{b}=0, \ldots, s_{b}, \quad r_{b}=0, \ldots, k_{b}, k_{c}=0, \ldots, s_{c}, r_{c}=0, \ldots, k_{c}\right)$ are the parameters that are being found during the problem solving.

The problem lies in image reconstructing of CT. In this, the accompanying is calculating of corresponding electrodynamic meshes and velocity fields.

We can reduce (1)-(5) to the series of more general boundary value problems on quasiconformal mapping $\omega=\omega^{(p)}(z)=\varphi^{(p)}(x, y)+i \psi^{(p)}(x, y)$ of the physical domains $G_{z}^{(p)}$ (Fig. 1, a) onto the corresponding domains of the complex quasipotential $G_{\omega}^{(p)}$ (Fig. 1, b) by the way, similarly to [4, 5, 14], of introducing the stream functions $\psi^{(p)}=\psi^{(p)}(x, y) \quad(p=1, \tilde{p})$, which are complex conjugated to $\varphi^{(p)}=\varphi^{(p)}(x, y) \quad(p=\overline{1, \tilde{p}})$, under (4) and (5) conditions:

$$
\begin{gathered}
\left\{\sigma_{11} \varphi_{x}^{\prime(p)}+\sigma_{12} \varphi_{y}^{\prime(p)}=\psi_{y}^{\prime(p)}, \sigma_{21} \varphi_{x}^{\prime(p)}+\sigma_{22} \varphi_{x}^{\prime(p)}=-\psi_{x}^{\prime(p)} ;\right. \\
\left.\varphi^{(p)}\right|_{A_{p} B_{p}}=\varphi_{*}^{(p)},\left.\varphi^{(p)}\right|_{C_{p} D_{p}}=\varphi^{*(p)}, \\
\left.\psi^{(p)}\right|_{A_{p} D_{p}}=0,\left.\quad \psi^{(p)}\right|_{B_{p} C_{p}}=Q^{(p)} ; \\
\int_{M N}\left|\vec{j}^{(p)}\right| d l=Q^{(p)}, M \in B_{p} C_{p}, N \in A_{p} D_{p} ; \\
\left.\varphi^{(p)}(M)\right|_{B_{p} C_{p}}=\bar{\varphi}^{(p)}(M),\left.\quad \varphi^{(p)}(M)\right|_{A_{p} D_{p}}=\underline{\varphi}^{(p)}(M), \\
\left.\psi^{(p)}(M)\right|_{A_{p} B_{p}}=\psi_{*}^{(p)}(M),\left.\quad \psi^{(p)}(M)\right|_{C_{p} D_{p}}=\psi^{*(p)}(M),
\end{gathered}
$$

where

$$
\begin{gathered}
\psi_{*}^{(p)}(M)=\int_{A_{p} M} \Psi_{*}^{(p)}(M) d l, \psi^{*(p)}(M)=\int_{D_{p} M} \Psi^{*(p)}(M) d l ; \\
G_{\omega}^{(p)}=\left\{(\varphi, \psi): \varphi_{*}^{(p)} \leq \varphi \leq \varphi^{*(p)}, 0 \leq \psi \leq Q^{(p)}\right\}
\end{gathered}
$$

$Q^{(p)}$ is discharge of the vector fields (current) through the contact sections $\left(A_{p} B_{p}\right.$ and $\left.C_{p} D_{p}\right) ; d l$ is arc element of corresponding curve.

The synthesis of the numerical quasiconformal mapping method and an idea of the rotational block parameterization. The most common approach for solving of the AQT forward problem is based on the using the finite element method. But the continuity conditions and, thus, the conserva- 
tion law for both a mesh cell and a whole domain doesn't satisfy [15]. So, we apply the finite difference method for discretization of functions and parameters of AQT mathematical model where the analogies of electrodynamic principle are considered. Also we apply quit modified algorithm for numerical solving of the boundary value problems on quasiconformal mapping of the domains with the different geometric configuration, which are limited by the stream and equiquasipotential lines [14] (including the advantages of the appropriate method) for the searching the unknown function of the current's potential $\varphi^{(p)}(x, y)$. We search the functions $\varphi^{(p)}=\varphi^{(p)}(x, y)$ and $\psi^{(p)}=\psi^{(p)}(x, y)$ when value $\left(\sigma_{\alpha \beta}(x, y, \ldots)\right)_{\alpha, \beta=1,2}$ is known, by the way of solving of the boundary value problems, inversed to (4)-(8), on quasiconformal mapping $G_{\omega}^{(p)} \rightarrow G_{z}^{(p)} \quad$ (relative to $x^{(p)}=x^{(p)}(\varphi, \psi) \quad$ and $\left.y^{(p)}=y^{(p)}(\varphi, \psi)\right)$ with statements which have the form [4, 5, 14]:

$$
\begin{aligned}
& \left\{\begin{array}{l}
\left(\frac{\sigma_{11} \sigma_{22}-\sigma_{21} \sigma_{12}}{\sigma_{11}} x_{\psi}^{\prime(p)}-\frac{\sigma_{21} x_{\varphi}^{\prime(p)}}{\sigma_{11}}\right)_{\psi}^{\prime}+\left(\frac{x_{\varphi}^{\prime(p)}}{\sigma_{11}}+\frac{\sigma_{12} x_{\psi}^{\prime(p)}}{\sigma_{11}}\right)_{\varphi}^{\prime}=0, \\
\left(\frac{\sigma_{11} \sigma_{22}-\sigma_{21} \sigma_{12}}{\sigma_{22}} y_{\psi}^{\prime(p)}+\frac{\sigma_{12} y_{\varphi}^{\prime(p)}}{\sigma_{22}}\right)_{\psi}^{\prime}+\left(\frac{y_{\varphi}^{\prime(p)}}{\sigma_{22}}-\frac{\sigma_{21} y_{\psi}^{\prime(p)}}{\sigma_{22}}\right)_{\varphi}^{\prime}=0 ;
\end{array}\right. \\
& x^{(p)}\left(\varphi_{*}^{(p)}, \psi\right)=\tilde{x}\left(\tau_{*}^{(p)}(\psi)\right), \quad y^{(p)}\left(\varphi_{*}^{(p)}, \psi\right)=\tilde{y}\left(\tau_{*}^{(p)}(\psi)\right), \\
& x^{(p)}\left(\varphi, Q^{(p)}\right)=\tilde{x}\left(\bar{\tau}^{(p)}(\varphi)\right), \quad y^{(p)}\left(\varphi, Q^{(p)}\right)=\tilde{y}\left(\bar{\tau}^{(p)}(\varphi)\right), \\
& x^{(p)}\left(\varphi^{*(p)}, \psi\right)=\tilde{x}\left(\tau^{*(p)}(\psi)\right), y^{(p)}\left(\varphi^{*(p)}, \psi\right)=\tilde{y}\left(\tau^{*(p)}(\psi)\right), \\
& x^{(p)}(\varphi, 0)=\tilde{x}\left(\underline{\tau}^{(p)}(\varphi)\right), y^{(p)}(\varphi, 0)=\tilde{y}\left(\underline{\tau}^{(p)}(\varphi)\right) ; \\
& \frac{y_{\varphi}^{\prime(p)}\left(\varphi_{*}^{(p)}, \psi_{*}^{(p)}\right)}{x_{\varphi}^{\prime(p)}\left(\varphi_{*}^{(p)}, \psi_{*}^{(p)}\right)}\left(\sigma_{11} y_{\psi}^{\prime(p)}\left(\varphi_{*}^{(p)}, \psi_{*}^{(p)}\right)-\sigma_{12} x_{\psi}^{\prime(p)}\left(\varphi_{*}^{(p)}, \psi_{*}^{(p)}\right)\right)= \\
& =\sigma_{21} y_{\psi}^{((p)}\left(\varphi_{*}^{(p)}, \psi_{*}^{(p)}\right)-\sigma_{22} x_{\psi}^{(p)}\left(\varphi_{*}^{(p)}, \psi_{*}^{(p)}\right), \tau \in\left[\tau_{B}^{(p)} ; \tau_{A}^{(p)}\right], \\
& \frac{y_{\varphi}^{\prime(p)}\left(\varphi^{*(p)}, \psi^{*(p)}\right)}{x_{\varphi}^{\prime(p)}\left(\varphi^{*(p)}, \psi^{*(p)}\right)}\left(\sigma_{11} y_{\psi}^{\prime(p)}\left(\varphi^{*(p)}, \psi^{*(p)}\right)-\sigma_{12} x_{\psi}^{\prime(p)}\left(\varphi^{*(p)}, \psi^{*(p)}\right)=\right. \\
& =\sigma_{21} y_{\psi}^{(p)}\left(\varphi^{*(p)}, \psi^{*(p)}\right)-\sigma_{22} x_{\psi}^{\prime(p)}\left(\varphi^{*(p)}, \psi^{*(p)}\right), \tau \in\left[\tau_{D}^{(p)} ; \tau_{C}^{(p)}\right], \\
& y_{\varphi}^{\prime(p)}\left(\underline{\varphi}^{(p)}, 0\right)\left(\sigma_{11} y_{\psi}^{\prime(p)}\left(\underline{\varphi}^{(p)}, 0\right)-\sigma_{12} x_{\psi}^{\prime(p)}\left(\underline{\varphi}^{(p)}, 0\right)\right)= \\
& =x_{\varphi}^{\prime(p)}\left(\underline{\varphi}^{(p)}, 0\right)\left(\sigma_{21} y_{\psi}^{\prime(p)}\left(\underline{\varphi}^{(p)}, 0\right)-\sigma_{22} x_{\psi}^{\prime(p)}\left(\underline{\varphi}^{(p)}, 0\right)\right), \tau \in\left[\tau_{A}^{(p)} ; \tau_{D}^{(p)}\right] \text {, }
\end{aligned}
$$




$$
\begin{aligned}
& \frac{y_{\varphi}^{\prime(p)}\left(\bar{\varphi}^{(p)}, Q^{(p)}\right)}{x_{\varphi}^{\prime(p)}\left(\bar{\varphi}^{(p)}, Q^{(p)}\right)}\left(\sigma_{11} y_{\psi}^{(p)}\left(\bar{\varphi}^{(p)}, Q^{(p)}\right)-\sigma_{12} x_{\psi}^{(p)}\left(\bar{\varphi}^{(p)}, Q^{(p)}\right)\right)= \\
& \quad=\sigma_{21} y_{\psi}^{\prime(p)}\left(\bar{\varphi}^{(p)}, Q^{(p)}\right)-\sigma_{22} x_{\psi}^{\prime(p)}\left(\bar{\varphi}^{(p)}, Q^{(p)}\right), \quad \tau \in\left[\tau_{C}^{(p)} ; \tau_{B}^{(p)}\right]
\end{aligned}
$$

under (4) and (5) conditions, where $\tau=\tau_{*}^{(p)}(\psi), \quad \tau=\bar{\tau}^{(p)}(\varphi)$, $\tau=\tau^{*(p)}(\psi), \quad \tau=\underline{\tau}^{(p)}(\varphi) \quad\left(\varphi_{*}^{(p)} \leq \varphi \leq \varphi^{*(p)}, \quad 0 \leq \psi \leq Q^{(p)}\right)$ - functions, which can be built by the way of interpolation of the experimentally received their values $\tau_{*_{j_{*}}}^{(p)}, \bar{\tau}_{\bar{i}}^{(p)}, \tau_{j^{*}}^{*(p)}, \underline{\tau}_{\underline{i}}^{(p)}$ having some arguments $\psi_{*_{j_{*}}}^{(p)}, \bar{\varphi}_{\bar{i}}^{(p)}$, $\psi_{j^{*}}^{*}(p), \underline{\varphi_{i}^{(p)}}$ on sections $A_{p} B_{p}, B_{p} C_{p}, C_{p} D_{p}$ and $A_{p} D_{p}$, respectively.

We reconstruct $\mathrm{CT}$, similarly to [3-5], using the minimizing functional of the sum of the squares of the residual of expressions, receiver from CauchyRiemann type conditions, (4) condition, within using regularization ideas

$$
\begin{gathered}
\Phi\left(x^{(1)}, \ldots, x^{(\tilde{p})}, y^{(1)}, \ldots, y^{(\tilde{p})}, a_{s_{a}, 0}, b_{s_{b}, 0}, c_{s_{c}, 0}, \ldots, a_{0, s_{a}}, b_{0, s_{b}}, c_{0, s_{c}}\right) \stackrel{d f}{=} \\
=\sum_{p=1}^{\tilde{p}}\left(\left(\sigma_{11} y_{\psi}^{\prime(p)}-\sigma_{12} x_{\psi}^{\prime(p)}-x_{\varphi}^{\prime(p)}+\sigma_{21} y_{\psi}^{\prime(p)}-\sigma_{22} x_{\psi}^{\prime(p)}-y_{\varphi}^{\prime(p)}\right)^{2}+\right. \\
+\left(\sigma_{11} y_{\psi}^{\prime(p)}-\sigma_{12} x_{\psi}^{\prime(p)}-x_{\varphi}^{\prime(p)}-\sigma_{21} y_{\psi}^{\prime(p)}+\sigma_{22} x_{\psi}^{\prime(p)}+y_{\varphi}^{(p)}\right)^{2}+ \\
\left.+2 \eta\left(\sum_{k_{a}=0}^{s_{a}} \sum_{r_{a}=0}^{k_{a}} \frac{a_{k_{a}-r_{a}, r_{a}}^{2}}{100^{k_{a}}}+\sum_{k_{b}=0}^{s_{b}} \sum_{r_{b}=0}^{k_{b}} \frac{b_{k_{b}-r_{b}, r_{b}}^{2}}{100^{k_{b}}}+\sum_{k_{c}=0}^{s_{c}} \sum_{r_{c}=0}^{k_{c}} \frac{c_{k_{c}}^{2}-r_{c}, r_{c}}{100^{k_{c}}}\right)\right)+ \\
+2 \mu\left(\lambda-\sigma_{11}-\sigma_{22}\right)^{2} \rightarrow \min
\end{gathered}
$$

and elliptic conditions [14]

$$
\sigma_{11} \sigma_{22}-\sigma_{12}^{2}>0, \sigma_{11}+\sigma_{22}>0
$$

where $\mu$ is multiplier, which affects the degree of implementation of the (4) condition, $\eta$ is regularizing parameter.

Let's write the difference analogues of problem (4), (5), (9)-(11) in mesh domains $G_{z}^{\gamma(p)}$, similarly to $[4,5,14]$, in such form:

$$
\left\{\begin{array}{l}
x_{i+1, j}^{(p)}+x_{i-1, j}^{(p)}-2\left(1+\gamma^{(p) 2} B_{i, j}^{(p)}\right) x_{i, j}^{(p)}+\gamma^{(p) 2} B_{i, j}^{(p)}\left(x_{i, j-1}^{(p)}+x_{i, j+1}^{(p)}\right)+\gamma^{(p)} \times \\
\times A_{i, j}^{(p)}\left(x_{i+1, j+1}^{(p)}+x_{i-1, j-1}^{(p)}-x_{i+1, j-1}^{(p)}-x_{i-1, j+1}^{(p)}\right) / 4+\left(x_{i, j+1}^{(p)}-x_{i, j-1}^{(p)}\right) \gamma^{(p)} D_{i, j}^{(p)}+ \\
+C_{i, j}^{(p)}\left(x_{i+1, j}^{(p)}-x_{i-1, j}^{(p)}\right)=0,0=y_{i+1, j}^{(p)}+y_{i-1, j}^{(p)}-2\left(1+\gamma^{(p) 2} B_{i, j}^{(p)}\right) y_{i, j}^{(p)}+ \\
+\gamma^{(p) 2} B_{i, j}^{(p)}\left(y_{i, j-1}^{(p)}+y_{i, j+1}^{(p)}\right)+\left(y_{i+1, j+1}^{(p)}+y_{i-1, j-1}^{(p)}-y_{i+1, j-1}^{(p)}-y_{i-1, j+1}^{(p)}\right) \gamma^{(p)} A_{i, j}^{(p)} / \\
/ 4+\left(y_{i, j+1}^{(p)}-y_{i, j-1}^{(p)}\right) \gamma^{(p)} F_{i, j}^{(p)}+E_{i, j}^{(p)}\left(y_{i+1, j}^{(p)}-y_{i-1, j}^{(p)}\right),\left(i=1, m^{(p)}, j=\overline{1, n^{(p)}}\right) ;
\end{array}\right.
$$




$$
\begin{aligned}
& x_{0, j}^{(p)}=\tilde{x}\left(\tau_{*}^{(p)}\left(\psi_{j}\right)\right), y_{0, j}^{(p)}=\tilde{y}\left(\tau_{*}^{(p)}\left(\psi_{j}\right)\right), x_{i, n^{(p)}+1}^{(p)}=\tilde{x}\left(\bar{\tau}^{(p)}\left(\varphi_{i}\right)\right), \\
& y_{i, n^{(p)}+1}^{(p)}=\tilde{y}\left(\bar{\tau}^{(p)}\left(\varphi_{i}\right)\right), x_{m^{(p)}+1, j}^{(p)}=\tilde{x}\left(\tau^{*(p)}\left(\psi_{j}\right)\right), \\
& y_{m^{(p)}+1, j}^{(p)}=\tilde{y}\left(\tau^{*(p)}\left(\psi_{j}\right)\right), x_{i, 0}^{(p)}=\tilde{x}\left(\underline{\tau}^{(p)}\left(\varphi_{i}\right)\right), \\
& y_{i, 0}^{(p)}=\tilde{y}\left(\underline{\tau}^{(p)}\left(\varphi_{i}\right)\right) \quad\left(0 \leq i \leq m^{(p)}+1, \quad 0 \leq j \leq n^{(p)}+1\right) ; \\
& \left(3 y_{0, j}^{(p)}-4 y_{1, j}^{(p)}+y_{2, j}^{(p)}\right)\left(\sigma_{11}\left(y_{0, j+1}^{(p)}-y_{0, j-1}^{(p)}\right)-\sigma_{12}\left(x_{0, j+1}^{(p)}-x_{0, j-1}^{(p)}\right)\right)= \\
& =\left(3 x_{0, j}^{(p)}-4 x_{1, j}^{(p)}+x_{2, j}^{(p)}\right)\left(\sigma_{21}\left(y_{0, j+1}^{(p)}-y_{0, j-1}^{(p)}\right)-\sigma_{22}\left(x_{0, j+1}^{(p)}-x_{0, j-1}^{(p)}\right)\right) \text {, } \\
& \left(3 y_{m^{(p)}+1, j}^{(p)}-4 y_{m^{(p)}, j}^{(p)}+y_{m^{(p)}-1, j}^{(p)}\right)\left(\sigma_{11}\left(y_{m^{(p)}+1, j+1}^{(p)}-y_{m^{(p)}+1, j-1}^{(p)}\right)-\right. \\
& \left.-\sigma_{12}\left(x_{m^{(p)}+1, j+1}^{(p)}-x_{m^{(p)}+1, j-1}^{(p)}\right)\right)=\left(\sigma_{21}\left(y_{m^{(p)}+1, j+1}^{(p)}-y_{m^{(p)}+1, j-1}^{(p)}\right)-\right. \\
& \left.-\sigma_{22}\left(x_{m^{(p)}+1, j+1}^{(p)}-x_{m^{(p)}+1, j-1}^{(p)}\right)\right)\left(3 x_{m^{(p)}+1, j}^{(p)}-4 x_{m^{(p)}, j}^{(p)}+x_{m^{(p)}-1, j}^{(p)}\right) \text {, } \\
& \left(y_{i+1,0}^{(p)}-y_{i-1,0}^{(p)}\right) \frac{\sigma_{11}\left(3 y_{i, 0}^{(p)}-4 y_{i, 1}^{(p)}+y_{i, 2}^{(p)}\right)-\sigma_{12}\left(3 x_{i, 0}^{(p)}-4 x_{i, 1}^{(p)}+x_{i, 2}^{(p)}\right)}{x_{i+1,0}^{(p)}-x_{i-1,0}^{(p)}}- \\
& -\left(\sigma_{21}\left(3 y_{i, 0}^{(p)}-4 y_{i, 1}^{(p)}+y_{i, 2}^{(p)}\right)-\sigma_{22}\left(3 x_{i, 0}^{(p)}-4 x_{i, 1}^{(p)}+x_{i, 2}^{(p)}\right)\right)=0, \\
& \left(\sigma_{11}\left(3 y_{i, n^{(p)}+1}^{(p)}-4 y_{i, n^{(p)}}^{(p)}+y_{i, n^{(p)}-1}^{(p)}\right)-\sigma_{12}\left(3 x_{i, n^{(p)}+1}^{(p)}-4 x_{i, n^{(p)}}^{(p)}+\right.\right. \\
& \left.\left.+x_{i, n^{(p)}-1}^{(p)}\right)\right)\left(y_{i+1, n^{(p)}+1}^{(p)}-y_{i-1, n^{(p)}+1}^{(p)}\right)-\left(\sigma _ { 2 1 } \left(3 y_{i, n^{(p)}+1}^{(p)}-4 y_{i, n^{(p)}}^{(p)}+\right.\right. \\
& \left.\left.+y_{i, n^{(p)}-1}^{(p)}\right)-\sigma_{22}\left(3 x_{i, n^{(p)}+1}^{(p)}-4 x_{i, n^{(p)}}^{(p)}+x_{i, n^{(p)}-1}^{(p)}\right)\right)\left(x_{i+1, n^{(p)}+1}^{(p)}-\right. \\
& \left.-x_{i-1, n^{(p)}+1}^{(p)}\right)=0, \quad i=\overline{0, m^{(p)}+1}, \quad j=\overline{0, n^{(p)}+1} \text {; } \\
& \lambda_{i, j}^{\gamma(p)}=\sigma_{11_{i, j}}^{\gamma(p)}+\sigma_{22_{i, j}}^{\gamma(p)}
\end{aligned}
$$

where $\gamma^{(p)}$ is quasiconformal invariant [14] for the corresponding domains

$$
\begin{gathered}
G_{\omega}^{\gamma(p)}=\left\{\left(\varphi_{i}^{(p)}, \psi_{j}^{(p)}\right): \varphi_{i}^{(p)}=\varphi_{*}^{(p)}+i \Delta \varphi^{(p)}, i=0, m^{(p)}+1 ;\right. \\
\psi_{j}^{(p)}=j \Delta \psi^{(p)}, j=0, n^{(p)}+1 ; \Delta \varphi^{(p)}=\left(\varphi^{*(p)}-\varphi_{*}^{(p)}\right) /\left(m^{(p)}+1\right), \\
\left.\Delta \psi^{(p)}=Q^{(p)} /\left(n^{(p)}+1\right), \gamma^{(p)}=\Delta \varphi^{(p)} / \Delta \psi^{(p)}, m^{(p)}, n^{(p)} \in \mathbf{N}\right\} ; \\
x_{i, j}^{(p)}=x^{(p)}\left(\varphi_{i}^{(p)}, \psi_{j}^{(p)}\right), \quad y_{i, j}^{(p)}=y^{(p)}\left(\varphi_{i}^{(p)}, \psi_{j}^{(p)}\right), \sigma_{\alpha \beta_{i, j}}^{\gamma(p)}=\sigma_{\alpha \beta}^{\gamma}\left(x_{i, j}^{(p)}, y_{i, j}^{(p)}\right), \\
\lambda_{i, j}^{\gamma(p)}=\lambda^{\gamma}\left(x_{i, j}^{(p)}, y_{i, j}^{(p)}\right) ; A_{i, j}^{(p)}, B_{i, j}^{(p)}, C_{i, j}^{(p)}, D_{i, j}^{(p)}, E_{i, j}^{(p)}, F_{i, j}^{(p)}
\end{gathered}
$$

are defined in the same way as [14]. 
We can rewrite the functional (12) and elliptic conditions (13) for all internal nodal points $\left(x_{i, j}^{(p)}, y_{i, j}^{(p)}\right) \quad\left(p=\overline{1, \tilde{p}}, \quad i=\overline{1, m^{(p)}}, \quad j=\overline{1, n^{(p)}}\right)$ as follows

$$
\begin{gathered}
\Phi\left(z_{1,1}^{(1)}, \ldots, z_{m^{(\tilde{p})}, n^{(\tilde{p})}}^{(\tilde{p})}, a_{s_{a}, 0}, b_{s_{b}, 0}, c_{s_{c}, 0}, \ldots, a_{0, s_{a}}, b_{0, s_{b}}, c_{0, s_{c}}\right)= \\
=\sum_{p, i, j=1}^{\tilde{p}, m^{(p)}, n^{(p)}}\left(\left(\frac{\sigma_{11_{i, j}}^{\gamma(p)}\left(y_{i, j+1}^{(p)}-y_{i, j-1}^{(p)}\right)-\sigma_{12_{i, j}}^{\gamma(p)}\left(x_{i, j+1}^{(p)}-x_{i, j-1}^{(p)}\right)}{\Delta \psi^{(p)}}-\right.\right. \\
\left.-\frac{x_{i+1, j}^{(p)}-x_{i-1, j}^{(p)}}{\Delta \varphi^{(p)}}\right)^{2}+\left(\frac{\sigma_{21_{i, j}}^{\gamma(p)}\left(y_{i, j+1}^{(p)}-y_{i, j-1}^{(p)}\right)-\sigma_{22_{i, j}}^{\gamma(p)}\left(x_{i, j+1}^{(p)}-x_{i, j-1}^{(p)}\right)}{\Delta \psi^{(p)}}-\right. \\
\left.-\frac{y_{i+1, j}^{(p)}-y_{i-1, j}^{(p)}}{\Delta \varphi^{(p)}}\right)^{2}+\eta\left(\sum_{k_{a}=0} \sum_{r_{a}=0}^{k_{a}} \frac{a_{k_{a}-r_{a}, r_{a}}^{2}}{100^{k_{a}}}+\sum_{k_{b}=0}^{s_{b}} \sum_{r_{b}=0}^{k_{b}} \frac{b_{k_{b}-r_{b}, r_{b}}^{2}}{100^{k_{b}}}+\right. \\
\left.\left.+\sum_{k_{c}=0}^{s_{c}} \sum_{r_{c}=0}^{k_{c}} \frac{c_{k_{c}-r_{c}, r_{c}}^{2}}{100^{k_{c}}}\right)+\mu\left(\lambda_{i, j}^{\gamma(p)}-\sigma_{11_{i, j}}^{\gamma(p)}-\sigma_{22_{i, j}}^{\gamma(p)}\right)^{2}\right) \rightarrow \min ; \\
\sigma_{11_{i, j}^{\gamma}}^{\gamma(p)} \sigma_{22_{i, j}^{\gamma}}^{\gamma(p)}-\sigma_{12_{i, j}}^{\gamma(p) 2} \geq \alpha_{1}, \sigma_{11_{i, j}}^{\gamma(p)}+\sigma_{22_{i, j}}^{\gamma(p)} \geq \alpha_{2}, \\
\sigma_{11_{i, j}}^{\gamma(p)}<\alpha_{3}, \sigma_{22_{i, j}}^{\gamma(p)}<\alpha_{4},
\end{gathered}
$$

where $\quad a_{k_{a}^{\prime}-r_{a}^{\prime}, r_{a}^{\prime}}, \quad b_{k_{b}^{\prime}-r_{b}^{\prime}, r_{b}^{\prime}}, \quad c_{k_{c}^{\prime}-r_{c}^{\prime}, r_{c}^{\prime}} \quad\left(k_{a}^{\prime}=\overline{0, s_{a}}, \quad r_{a}^{\prime}=\overline{0, k_{a}^{\prime}}, \quad k_{b}^{\prime}=\overline{0, s_{b}}\right.$, $\left.r_{b}^{\prime}=\overline{0, k_{b}^{\prime}}, \quad k_{c}^{\prime}=\overline{0, s_{c}}, \quad r_{c}^{\prime}=\overline{0, k_{c}^{\prime}}\right)$ are the desired parameters. The solving of the nonlinear programming problem (18)-(19) is expedient to implement by one of the appropriate methods of local optimization (for example, by the penalty functions method [16]).

Conclusions. The methodology $[4,5]$ of image reconstruction based on the idea of the quasiconformal similarity in the small of building the curvilinear quadrangles - dynamical mesh components in the physical domain and the corresponding rectangles in the complex quasipotential domain using AQT data and the rotational parameterization of internal nodes of dynamic meshes (which are built for each injections) and searched CT is generalized to the case of anisotropic media. We set the liquid (current) distribution and the averaged potential at the contact sections of plate and body unlike the traditional approaches to the statement and solving the problems of electrical impedance tomography [1-3, 6-13].

We plan to extend the proposed algorithm to the following cases: spatial resolution, applying the quasipotential of the initial stream to several sections, parameters identification of CT for piecewise-homogeneous and piecewise- 
inhomogeneous media (which, in particular, have place in medical diagnostics). Moreover, in medical practice there are media where the dependence of conductivity from the direction is not necessarily determined by the corresponding ellipse. In this case, it is expedient to represent the $\mathrm{CT}$ as a complex function. This is one of the areas for our future researches too.

\section{References:}

1. Holder D. Electrical Impedance Tomography. Methods, History and Applications / D. Holder. - Bristol : IOP Publishing, 2005. — 456 p.

2. Horb M. S. Choice of the study object for mathematical model in electrical impedance tomography / M. S. Horb, O. V. Husieva // Bulletin of NTUU «KPI». Radiotechnique, Radioaparatus Building Series. - Kyiv: NTUU «KPI», 2013. - Vol. 52. - P. 120-128. (Ukr).

3. Sherina E. S. Finite volume schemes for the electrical impedance tomography problem / E. S. Sherina, A. V. Starchenko // Tomsk State University Journal of Mathematics and Mechanics. — 2014. - Vol. 29 (3). - P. 25-38. (Rus).

4. Bomba A. Ya. Numerical Methods of Quasiconformal Mappings for Solving Problems of Identifying of Electrical Conductivity Coefficient in an Applied Potential Tomography / A. Ya. Bomba, L. L. Kroka// Volyn Mathematical Bulletin. Applied Mathematics Series. - Rivne : Editorial and Publishing Department of RSHU, 2014. - Vol. 11 (20). - P. 24-33. (Ukr).

5. Bomba A. Ya. Applied Quasipotenrial Method for Solving Coefficient Problems of Parametric Identification / A. Ya. Bomba, M. V. Boichura // Bulletin of NUWEE. Technical Sciences Series. - Rivne : Editorial and Publishing Department of NUWEE, 2017. - Vol. 4 (76). - P. 163-177. (Ukr).

6. Martins T. C. Investigating Anisotropic EIT with Simulated Annealing / T. C. Martins, M. S. G. Tsuzuki // IFAC-PapersOnLine. — 2017. — Vol. 50 (1). P. 9961-9966.

7. Lionheart W. R. B. Conformal uniqueness results in anisotropic electrical impedance imaging Inverse Problems / W. R. B. Lionheart// Inverse Problems. - 1997. - Vol. 13 (1). - P. 125-134.

8. Electrical impedance tomography in anisotropic media with known eigenvectors / J.-F. P. J. Abascal, W. R. B. Lionheart, S. R. Arridge and other // Inverse Problems. - 2011. - Vol. 27 (6). - P. 1-17.

9. Anisotropic resistivity tomography / J. V. Herwanger, C. C. Pain, A. Binley and other // Geophysical Journal International. — 2004. — Vol. 158 (2). P. 409-425.

10. Crabb M. EIT Reconstruction Algorithms for Respiratory Intensive Care. PhD Thesis / M. Crabb // Manchester :University of Manchester. - 2014.

11. Tallman T. N. Conductivity-Based Nanocomposite Structural Health Monitoring via Electrical Impedance Tomography. PhD Thesis / T. N. Tallman // Ann Arbor : University of Michigan. - 2015.

12. Use of anisotropic modelling in electrical impedance tomography; Description of method and preliminary assessment of utility in imaging brain function in the adult human head / J.-F. P. J. Abascal, S. R. Arridge, D. Atkinson and other // NeuroImage. — 2008. - Vol. 43 (2). - P. 258-268. 
13. Vassilevski Yu. V. Finite element analysis in bioimpedance diagnostics / Yu. V. Vassilevski, A. A. Danilov, D. V. Nikolaev, A. V. Smirnov // Computational Mathematics and Mathematical Physics. - 2012. - Vol. 52 (4). P. 733-745 (Rus).

14. Bomba A. Ya. Complex analysis methods / A. Ya. Bomba, S. S. Kashtan, D. O. Pryhornytskyi, S. V. Yaroshchak. - Rivne : Editorial and Publishing Department of NUWEE, 2013. - 415 p. (Ukr).

15. The comparison between FVM and FEM for EIT forward problem / G. Dong, J. Zou, R. Bayford and other// IEEE Transactions on Magnetics. - 2005. Vol. 41 (5). - P. 1468-1471.

16. ALGLIB - $\mathrm{C}++/ \mathrm{C} \#$ numerical analysis library. URL: http://www.alglib.net (last accessed 2018/11/06).

\section{ЧИСЛОВИЙ МЕТОД КОМПЛЕКСНОГО АНАЛІЗУ ІДЕНТИФІКАЦІЇ ПАРАМЕТРІВ АНІЗОТРОПНИХ СЕРЕДОВИЩ ЗА ДАНИМИ ТОМОГРАФІЇ ПРИКЛАДЕНИХ КВАЗІПОТЕНЦІАЛІВ. ЧАСТИНА 1: ПОСТАНОВКА ТА НАБЛИЖЕННЯ ЗАДАЧІ}

Підхід до розв'язання градієнтних задач ідентифікації параметрів квазіідеальних полів за даними томографії прикладених квазіпотенціалів на основі числових методів комплексного аналізу перенесено на випадки анізотропних середовищ. При цьому, аналогічно до існуючих робіт світових вчених, апріорно відомими вважаються деякі додаткові відомості про характер розподілу провідності всередині області (об'єкта дослідження). Проте, на відміну від традиційних підходів до постановки та розв'язання задач електроімпедансної томографії, на ділянках контакту пластинки і тіла окрім усередненого потенціалу тут задається ще й розподіл локальних швидкостей речовини (рідини, струму), а на інших ділянках (лініях течіі) - розподіл потенціалу (за експериментальними даними, які апроксимуємо із застосуванням сплайнів, кривих Безьє тощо). Генерація вихідних даних на межі досліджуваного об'єкта здійснюється відповідно до полярної моделі (схеми) інжекції струму при заданій сумі власних значень тензора провідності середовища. Наявність такого виду даних значно пришвидшує процес подальшого розв'язання поставленої задачі, що зручно, зокрема, при верифікації розробленого авторами методу. Відповідна задача зводиться до ітераційного розв'язання серії задач для рівнянь типу Лапласа, де замість «приграничних числових аналогів рівнянь типу Коші-Рімана» фігурують співвідношення квазіортогональності за спеціальних типів умов оптимізації. А саме: мінімізуючий функціонал побудований з урахуванням умов типу Коші-Рімана, співвідношення між відповідними тензору анізотропії власними значеннями, а також регуляризуючого доданку; умови-обмеження сформовані на основі умов еліптичності.

Ключові слова: томографія прикладених квазіпотениіалів, квазіконформні відображення, анізотропія, ідентифікачія, нелінійні задачі.

Отримано: 28.11.2018 